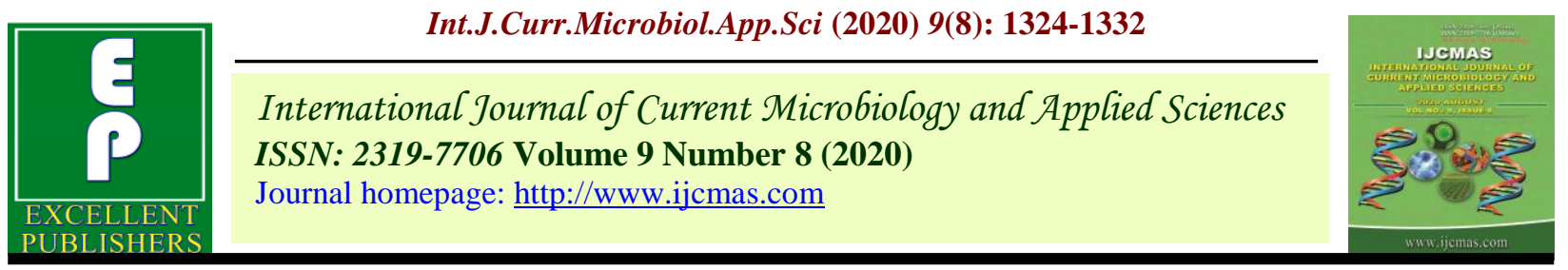

Original Research Article

https://doi.org/10.20546/ijcmas.2020.908.150

\title{
Stability Analysis of Pistillate x Pistillate based Hybrids and their Parents for Seed Yield in Castor (Ricinus communis $\mathbf{L}$.
}

\author{
A. R. Aher*, M. S. Kamble, M. S. Mote, A. G. Bhoite and S. R. Shinde \\ Agricultural Botany Division, RCSM College of Agriculture, Kolhapur 416004 \\ (University: Mahatma Phule Krishi Vidyapeeth, Rahuri, Maharashtra), India \\ *Corresponding author
}

\begin{tabular}{|l|} 
Ke y w o r d s \\
Ricinus communis \\
$\begin{array}{l}\text { L., Pistillate x } \\
\text { pistillate } \mathrm{G} \text { x } \\
\text { interaction, Stability }\end{array}$ \\
\hline Article Info \\
\hline $\begin{array}{l}\text { Accepted: } \\
15 \text { July } 2020 \\
\text { Available Online: } \\
10 \text { August } 2020\end{array}$ \\
\hline
\end{tabular}

A B S T R A C T

Thirty six pistillate $\mathrm{x}$ pistillate base hybrids, nine parents and one commercial check were evaluated under three environments in randomized complete block design with three replications. Significant differences were observed for genotype, environments and genotype $\mathrm{x}$ environment interaction. Stability analysis revealed that none of the genotypes was found consistently stable for all five characters in any environment. Base on mean, regression coefficient (bi) and deviation from regression $\left(\mathrm{S}^{2} \mathrm{di}\right)$ the parent ANDCP-06-07 as well as hybrids ANDCP-08-01 x JP-65, ANDCP-06-07 x ACP-1-06-07, ANDCP-06-07 x DPC-9, VP-1 x DPC-9 and DPC-9 $x$ ANDCP-06-07-1 had average stability and wider adaptability; whereas, parents ACP-1-06-07, SKP-84, ANDCP-06-07-1 and hybrid ACP-1-06-07 x JP-65 had above average stability and well adapted to poor environment, similarly hybrids ANDCP-08-01 x ANDCP-06-07-1, ACP06-07-1 x DPC-9, GCH-7 (Check) had below average stability and specifically adapted to favorable environment for seed yield per plant.

\section{Introduction}

Castor is grown in tropical, sub-tropical and temperate regions of the world. It is cultivated in about 30 countries on commercial scale. Among those, India, Brazil, China, Russia, Thailand and Philippines are the principal castor growing countries. Being the largest producer, India is also largest exporter of castor seed oil and exports $80 \%$ of its total castor oil to China, which is the world's largest importer of castor oil followed by US, Japan, Thailand and other European countries. Gujarat is leading castor growing state, where the crop was grown in around 8.72 lakh ha. with 17.88 lakh tonnes production and productivity of $2050 \mathrm{~kg} / \mathrm{ha}$ (Anno, 2019). 
Development and adaption of high yielding genotypes under wider range of diversified environments is one of the major goals for the plant breeders in castor improvement programme. Therefore, the present study of the genotype $\mathrm{x}$ environment interaction is important in $F_{1}$ generation is that the $F_{1} S$, which are stable in varied environments, are likely to produce stable segregants in succeeding generations, and those may be looked for selection of desirable genotypes. Generally multilocation trials are conducted for the several years to find out stability. However, economy could be exercised by manipulating agronomic differentials like sowing dates, plant geometry, doses of fertilizer, irrigations, spacing etc. at a single sowing location and season. In order to minimize genotypes $\mathrm{x}$ environments interactions, and to increase precision in selection, stratification of environments has been employed; however, even with refinement of technique, an interaction of genotypes with environments within same year remains very large (Allard and Bradshaw, 1964).

The testing of genotypes over environments proves an opportunity to study the adaptability of a genotype to a particular environment and also the stability of a genotype over different environments. Precise knowledge on the nature and magnitude of genotype $\mathrm{x}$ environment interactions is important in understanding the stability in yield of a particular variety or hybrid before it is being recommended for a given situation. The present study was undertaken to identify stable pistillate $\mathrm{x}$ pistillate hybrids and their parents for seed yield and four component characters.

\section{Materials and Methods}

The experimental material consisted of nine genetically diverse pistillate lines viz.
ANDCP-08-01, ANDCP-06-07, ACP-1-0607, SKP-84, VP-1, DPC-9, JP-65, ANDCP06-07-1 and ANDCP-06-07-2 were crossed in half diallel mating fashion. The resulting 46 genotypes (36 hybrids +9 parents $+\mathrm{GCH}-7$ as commercial check) were grown in Randomized Complete Block Design with three replications in three environments viz., E1 (Late kharif - Second week of September; $120 \times 60 \mathrm{~cm}^{2}$ ), E2 (Autumn - Second week of October; 90 × $60 \mathrm{~cm}^{2}$ ) and E3 (Rabi- First week of November; 90 x $45 \mathrm{~cm}^{2}$ ). The investigation was carried out at Regional Research Station, Anand Agricultural University, Anand. All recommended package of practices were followed for good crop stand and growth. Five competitive plants in each replication were randomly selected for recording seed yield per plant, plant height up to base of primary raceme and number of nodes up to base of primary raceme. Whereas, data on days to $50 \%$ flowering of primary raceme and days to 50 $\%$ maturity of primary raceme were recorded on plot basis. The genotype $\mathrm{x}$ environment interaction and stability parameters were estimated as per the model of Eberhart and Russell (1966).

\section{Results and Discussion}

The results of the combined analysis of variance as per Eberhart and Russell model for seed yield per plant, plant height up to base of primary raceme, number of nodes up to base of primary raceme, days to $50 \%$ flowering of primary raceme and days to 50 $\%$ maturity of primary raceme are presented in table 1 . The highly significant values of mean square due to genotypes, genotype $\mathrm{x}$ environment and environments (linear) for all the characters indicated that environments differed considerably among different sowing dates. This suggesting the existence of considerable variation among the genotypes as well as created environment. Similar 
finding were also reported by Solanki and Joshi (2003) and Patel (2009). The mean square values due to $\mathrm{G} X \mathrm{E}$ (linear) and pooled deviation were found to be significant for the all the characters. Significant $G \times$ E interactions (linear) for seed yield per plant were earlier reported by Solanki and Joshi (2003), Sasidharan (2005), Chaudhari (2006) and Patel (2009). An evaluation of genotype environment interaction gives an idea of the buffering capacity of the genotypes under study; the low magnitude of genotype environment interactions indicates consistent performance of genotypes over environments for particular characters. Both relative magnitude of linear and non-linear components of $\mathrm{G} X \mathrm{E}$ interaction would decide, whether the performance of a genotype for the character under consideration would be predictable or not. Since, when both linear and non-linear (pooled deviation) components of $\mathrm{G} X \mathrm{E}$ interaction are significant, the magnitude of both the components need to be considered, and greater magnitude of linear component suggests possibility for prediction of performance of genotypes over environments.

Environmental index directly reflects the poor or rich environment in terms of negative and positive index, respectively. The higher seed yield per plant was obtained under Autumn season (Better environment); whereas, lower seed yield per plant was obtained under late Kharif and Rabi seasons, which were considered as poor environments. For plant height up to base of primary raceme, days to $50 \%$ flowering of primary raceme and days to $50 \%$ maturity of primary raceme the rabi season was considered as better environment and Autumn and late Kharif season was considered as poor environment. Likewise, for number of nodes up to base of primary raceme the late Kharif season was considered as better environment and Autumn and rabi season was considered as poor environment. None of the environment was found consistently better for all the characters (Table 2).

The estimate of mean performance (x), regression coefficient (bi) and deviation from regression $\left(S^{2} \mathrm{di}\right)$ presented in Table 3 to 5 . The stability parameters employed for identification of stable genotypes were high or low mean values than population mean as the character has economic importance, a regression coefficient (bi) equals to unity and a mean square deviation from linear regression coefficient statistically equal to zero $\left(S^{2} d_{i}\right)$. For seed cotton yield per plant 13 genotypes were identified as well adapted to different environments. Among the parental genotypes, parent ANDCP-06-07 had average stability $\quad\left(\right.$ Mean $<$ parental mean; $b_{i}=0$ significant and $\left.\mathrm{b}_{\mathrm{i}}=1 \quad \mathrm{NS} ; \quad \mathrm{S}^{2} \mathrm{~d}_{\mathrm{i}}=0 \quad \mathrm{NS}\right)$, suggesting it as widely adapted to all the environments. Parents ACP-1-06-07, SKP-84 and ANDCP-06-07-1 had above average stability (Mean>parental mean; $b_{i}=0$ significant; $b_{i}=1$ significant and $b_{i}<1.00$; $\mathrm{S}^{2} \mathrm{~d}_{\mathrm{i}}=0 \mathrm{NS}$ ), thereby specifically adapted to poor environment. Among the hybrids, hybridsANDCP-08-01 x JP-65, ANDCP-06$07 \times$ ACP-1-06-07, ANDCP-06-07 x DPC-9, VP-1 x DPC-9 and DPC-9 x ANDCP-06-07-1 were found stable and widely adapted to all the environments (Mean>hybrids mean; $b_{i}=0$ significant and $b_{i}=1$ non significant; $S^{2} d_{i}=0$ NS), while hybrids ANDCP-08-01 $\mathrm{x}$ ANDCP-06-07-1,ACP-06-07-1 x DPC-9, and GCH-7 (Check) had below average stability (Mean >hybrids mean; $b_{i}=0$ significant; $b_{i}=1$ significant and $b_{i}>1.00 ; S^{2} d_{i}=0 \mathrm{NS}$ ), thereby well adapted to favorable environment. On the other hand, hybrid ACP-1-06-07 x JP-65 had above average stability (Mean $>$ hybrids mean; $b_{i}=0$ significant; $b_{i}=1$ Significant and $\mathrm{b}_{\mathrm{i}}<1.00 ; \mathrm{S}^{2} \mathrm{~d}_{\mathrm{i}}=0 \mathrm{NS}$ ), and specifically adapted to unfavorable or poor environment.

For pistillate parent dwarf plant stature is desirable. The parental mean and hybrids mean were 53.55 and $63.22 \mathrm{~cm}$, respectively. 
The results revealed that pistillate parent SKP-84 had average stability; whereas, two pistillate parents VP-1 and ANDCP-06-07-1 had above average stability, revealing them specifically adapted to poor environment. Among the hybrids, ANDCP-06-07 x SKP-84 and SKP-84 $x$ ANDCP-06-07-2 had average stability; whereas, four hybrids ANDCP-0801 x ACP-1-06-07, ANDCP-06-07 xANDCP06-07-2, ANDCP-06-07 x ANDCP-06-07-2 and SKP-84 x ANDCP-06-07-1 had below average stability, and well adapted to favorable environment, while four hybrids ANDCP-06-07 x ACP-1-06-07, ACP-1-06-07 $x$ ANDCP-06-07-1, ACP-1-06-07 x ANDCP06-07-2, and VP-1 x ANDCP-06-07-2 had above average stability, thereby specifically adapted to poor environment.

The character number of nodes up to base of primary raceme is positively correlated with plant height, and for pistillate parent requirement is for dwarf plant stature, hence genotypes with minimum number of nodes up to base of primary raceme are favoured. The pistillate parent ANDCP-06-07-1 and hybrids ANDCP-08-01 x VP-1, ANDCP-08-01 $x$ ANDCP-06-07-1, ANDCP-06-07 x ANDCP06-07-1, ACP-1-06-07 x DPC-9 and VP-1 x JP-65 had average stability. Whereas two hybrids VP-1 x ANDCP-06-07-1 and VP-1 x ANDCP-06-07-2 had below average stability; hence, specifically adapted to favorable environment, and four hybrids ANDCP-08-01 x SKP-84, ANDCP-08-01 x JP-65, ANDCP06-07 x JP-65 and DPC-9 x ANDCP-06-07-1 had above average stability, thereby specifically adapted to poor environment.

Earliness is prerequisite for any crop species in climate change, hence minimum days to $50 \%$ flowering and maturity of primary raceme are desirable. For days to $50 \%$ flowering of primary raceme the parental genotypes, ANDCP-06-07, SKP-84 and
ANDCP-06-07-2 had average stability, whereas parents VP-1 and ANDCP-06-07-1 had above average stability specifically adapted to poor environment. Among hybrids, nine hybrids ANDCP-08-01 x ANDCP-0607,ANDCP-08-01 x SKP-84, ANDCP-08-01 x DPC-9, ANDCP-06-07 x DPC-9, ANDCP06-07 x JP-65, ANDCP-06-07 x ANDCP-0607-1, ACP-1-06-07 x VP-1, SKP-84x ANDCP-06-07-1, SKP-84 x ANDCP-06-07-2 and $\mathrm{GCH}-7$ (Check) had average stability; while two hybrids ANDCP-06-07 x ANDCP06-07-2 and SKP-84 x DPC-9 had below average stability; whereas, six hybrids ANDCP-08-01 x ACP-1-06-07, ANDCP-0801 x JP-65, ANDCP-06-07 x VP-1, ACP-106-07 x DPC-9, VP-1 x ANDCP-06-07-1 and VP-1 x ANDCP-06-07-2 had above average stability, thereby specifically adapted to poor environment.

For days to $50 \%$ maturity of primary raceme the pistillate parents, parents ANDCP-06-07, DPC-9 and ANDCP-06-07-1 had below average stability, thereby adapted to better environment. Whereas, two pistillate parents SKP-84 and VP-1 had above average stability, hence specifically adapted to poor environment. Among the hybrids, hybrids ANDCP-06-07 x ANDCP-06-07-2, ACP-106-07 x DPC-9, SKP-84 x DPC-9, SKP-84 x ANDCP-06-07-1, SKP-84x ANDCP-06-07-2, VP-1 $\mathrm{x}$ ANDCP-06-07-1 and VP-1 $\mathrm{x}$ ANDCP-06-07-2 had average stability, and these hybrids would have wide adaptation; whereas, hybrids ANDCP-08-01 x DPC-9, ANDCP-06-07 x VP-1, VP-1 xJP-65 and ANDCP-06-07-1 $\mathrm{x}$ ANDCP-06-07-2had below average stability and hybrids ANDCP08-01 x ANDCP-06-07, ANDCP-08-01 x SKP-84, ACP-1-06-07 x VP-1, and DPC-9 x ANDCP-06-07-2 had above average stability (Mean $<$ hybrids mean; $b_{i}=0$ significant; $b_{i}=1$ significant and $b_{i}<1.00 ; S^{2} d_{i}=0 \mathrm{NS}$ ); thereby specifically adapted to poor environment. 
Table.1 Analysis of variance for phenotypic stability for different characters in castor

\begin{tabular}{|c|c|c|c|c|c|c|c|}
\hline \multirow{2}{*}{$\begin{array}{c}\text { Source } \\
\text { Rep. within Env. }\end{array}$} & \multirow{2}{*}{$\begin{array}{l}\text { df } \\
6\end{array}$} & \multicolumn{2}{|c|}{ Seed yield per plant } & \multicolumn{2}{|c|}{$\begin{array}{c}\text { Plant height up to base of } \\
\text { primary raceme }\end{array}$} & \multicolumn{2}{|c|}{$\begin{array}{c}\text { Number of nodes up to base } \\
\text { of primary raceme }\end{array}$} \\
\hline & & 497.994 & & 32.25 & $*$ & 3.086 & $\# * *$ \\
\hline Genotypes & 45 & 16320.820 & @@ \#\#** & 1965.236 & @@ \#\#** & 8.231 & @@ \#\#** \\
\hline Environments & 2 & 219969.500 & @@ \#\#** & 503.359 & @@ \#\#** & 112.176 & @@ \#\#** \\
\hline Geno. x Env. & 90 & 4846.68 & $* *$ & 53.585 & $* *$ & 1.688 & $\# * *$ \\
\hline Env. + (Geno. $x$ Env. $)$ & 92 & 9523.264 & $\# \# * *$ & 63.363 & $\# * *$ & 4.090 & $\# \# * *$ \\
\hline Environments (Lin.) & 1 & 439939.100 & $\# \# * *$ & 1006.717 & \#\# ** & 224.351 & $\# \# * *$ \\
\hline Geno. x Env. (Lin.) & 45 & 6019.572 & $\# * *$ & 68.470 & $\# * *$ & 2.296 & $\# \# * *$ \\
\hline Pooled deviation & 46 & 3593.923 & $* *$ & 37.860 & $* *$ & 1.057 & $* *$ \\
\hline Pooled error & 270 & 457.154 & & 12.227 & & 0.545 & \\
\hline Total & & 11756.04 & & 688.066 & & 5.45 & \\
\hline
\end{tabular}

\begin{tabular}{|l|c|c|c|c|c|}
\hline \multicolumn{1}{|c|}{ Source } & df & \multicolumn{2}{|c|}{$\begin{array}{c}\text { Days to 50 \% flowering of } \\
\text { primary raceme }\end{array}$} & \multicolumn{2}{c|}{$\begin{array}{c}\text { Days to 50 \% maturity } \\
\text { of primary raceme }\end{array}$} \\
\hline Rep. within Env. & 6 & 49.225 & @ @ \#\#** & 23.589 & $* *$ \\
\hline Genotypes & 45 & 58.698 & @ @ \#\#** & 133.981 & @ @ \#\#** \\
\hline Environments & 2 & 5615.406 & @ @ \#\#** & 4527.781 & @ @ \#\#** \\
\hline Geno. x Env. & 90 & 10.897 & $* *$ & 20.000 & $* *$ \\
\hline Env. + (Geno. x Env.) & 92 & 132.734 & $\# \# * *$ & 117.995 & \#\#** \\
\hline Environments (Lin.) & 1 & 11230.810 & $\# \# * *$ & 9055.562 & \#\#** \\
\hline Geno. x Env. (Lin.) & 45 & 13.866 & $\# * *$ & 23.817 & $* *$ \\
\hline Pooled deviation & 46 & 7.756 & $* *$ & 15.831 & $* *$ \\
\hline Pooled error & 270 & 4.644 & & 6.561 & \\
\hline Total & & 108.416 & & 123.246 & \\
\hline
\end{tabular}

@, @ @ Significant tested against genotypes x environments (G x E) at 0.05 and 0.01 levels of probability, respectively \# \#\# Significant tested against pooled deviation at 0.05 and 0.01 levels of probability, respectively

*, ** Significant tested against pooled error at 0.05 and 0.01 levels of probability, respectively

Table.2 Environmental index (I) for different quantitative characters

\begin{tabular}{|c|l|r|r|r|}
\hline SN & Characters & \multicolumn{1}{|c|}{$\mathbf{I}_{\mathbf{1}}$} & \multicolumn{1}{|c|}{$\mathbf{I}_{\mathbf{2}}$} & \multicolumn{1}{|c|}{$\mathbf{I}_{\mathbf{3}}$} \\
\hline $\mathbf{1 .}$ & Seed yield per plant & -38.799 & 79.839 & -41.040 \\
\hline $\mathbf{2 .}$ & Plant height up to primary raceme & -1.461 & -2.326 & 3.787 \\
\hline $\mathbf{3 .}$ & Number of nodes up to primary raceme & 1.802 & -0.947 & -0.856 \\
\hline $\mathbf{4 .}$ & Days to 50\% flowering of primary raceme & -8.328 & -4.205 & 12.534 \\
\hline $\mathbf{5 .}$ & Days to 50\% maturity of primary raceme & -7.614 & -3.606 & 11.220 \\
\hline
\end{tabular}

$I_{1}, I_{2}$ and $I_{3}$ environmental index for environments $E_{1}, E_{2}$ and $E_{3}$, respectively.

Table.3 Stability parameters for seed yield per plant

\begin{tabular}{|r|l|r|r|r|r|}
\hline \multicolumn{1}{|c|}{ S.N } & \multicolumn{1}{|c|}{ Parents / Crosses } & Code & \multicolumn{3}{c|}{ Seed yield per plant } \\
\cline { 4 - 7 } $\mathbf{0}$ & & & Mean & $\mathbf{b}_{\mathbf{i}}$ & \multicolumn{1}{c|}{$\mathbf{S}^{\mathbf{2}} \mathbf{d}_{\mathbf{i}}$} \\
\hline $\mathbf{1}$ & ANDCP-08-01 & P1 & 116.79 & $0.84 * * @$ & -410.73 \\
\hline $\mathbf{2}$ & ANDCP-06-07 & P2 & 174.98 & $0.23 * *$ & 167.80 \\
\hline $\mathbf{3}$ & ACP-1-06-07 & P3 & 169.62 & $0.51 * * @ @$ & -194.79 \\
\hline $\mathbf{4}$ & SKP-84 & P4 & 195.21 & $0.78 * * @ @$ & -436.59 \\
\hline $\mathbf{5}$ & VP-1 & P5 & 196.18 & $1.27 *$ & 2384.78 \\
\hline
\end{tabular}




\begin{tabular}{|c|c|c|c|c|c|c|c|}
\hline 6 & DPC-9 & P6 & 128.72 & -0.04 & & 5944.63 & $\#$ \\
\hline 7 & JP-65 & P7 & 135.49 & -0.53 & & 25133.88 & $\#$ \\
\hline 8 & ANDCP-06-07-1 & P8 & 193.29 & 0.71 & **@ & -320.66 & \\
\hline \multirow[t]{2}{*}{9} & ANDCP-06-07-2 & P9 & 167.75 & 0.53 & & 3412.96 & $\#$ \\
\hline & Parental Mean & & 164.22 & & & & \\
\hline 10 & ANDCP-08-01 x ANDCP-06-07 & $\mathrm{P} 1 \times \mathrm{P} 2$ & 211.90 & 1.09 & $*$ & 2123.99 & $\#$ \\
\hline 11 & ANDCP-08-01 x ACP-1-06-07 & $\mathrm{P} 1 \times \mathrm{P} 3$ & 208.51 & 0.38 & @ & 319.31 & \\
\hline 12 & ANDCP-08-01 x SKP-84 & $\mathrm{P} 1 \times \mathrm{P} 4$ & 197.41 & 0.08 & & 5723.93 & $\#$ \\
\hline 13 & ANDCP-08-01 x VP-1 & P1 x P5 & 203.90 & -0.06 & & 4967.29 & $\#$ \\
\hline 14 & ANDCP-08-01 x DPC-9 & P1 x P6 & 223.87 & 1.38 & & 5516.22 & $\#$ \\
\hline 15 & ANDCP-08-01 x JP-65 & P1 x P7 & 366.42 & 0.93 & $* *$ & -335.58 & \\
\hline 16 & ANDCP-08-01 x ANDCP-06-07-1 & $\mathrm{P} 1 \times \mathrm{P} 8$ & 262.16 & 2.10 & **@@ & 365.54 & \\
\hline 17 & ANDCP-08-01 x ANDCP-06-07-2 & P1 x P9 & 221.82 & 1.36 & & 5928.43 & $\#$ \\
\hline 18 & ANDCP-06-07 x ACP-1-06-07 & $\mathrm{P} 2 \times \mathrm{P} 3$ & 264.05 & 0.95 & $* *$ & -435.09 & \\
\hline 19 & ANDCP-06-07 x SKP-84 & $\mathrm{P} 2 \times \mathrm{P} 4$ & 210.51 & 0.13 & @@ & 570.35 & \\
\hline 20 & ANDCP-06-07 x VP-1 & $\mathrm{P} 2 \times \mathrm{P} 5$ & 172.55 & 0.11 & @ & 1040.92 & \\
\hline 21 & ANDCP-06-07 x DPC-9 & $\mathrm{P} 2 \times \mathrm{P} 6$ & 308.72 & 1.51 & $* *$ & 319.95 & \\
\hline 22 & ANDCP-06-07 x JP-65 & $\mathrm{P} 2 \times \mathrm{P} 7$ & 371.17 & 1.32 & & 4116.71 & $\#$ \\
\hline 23 & ANDCP-06-07 x ANDCP-06-07-1 & $\mathrm{P} 2 \times \mathrm{P} 8$ & 128.64 & 0.22 & *@@ & -338.168 & \\
\hline 24 & ANDCP-06-07 x ANDCP-06-07-2 & $\mathrm{P} 2 \times \mathrm{P} 9$ & 172.32 & 0.69 & & 1003.87 & \\
\hline 25 & ACP-1-06-07 x SKP-84 & $\mathrm{P} 3 \times \mathrm{P} 4$ & 211.85 & 0.17 & & 11997.35 & \# \\
\hline 26 & ACP-1-06-07 x VP-1 & P3 x P5 & 209.41 & 1.25 & $* *$ & 455.32 & \\
\hline 27 & ACP-1-06-07 x DPC-9 & P3 x P6 & 329.93 & 2.14 & **@@@ & -381.82 & \\
\hline 28 & ACP-1-06-07 x JP-65 & P3 x P7 & 297.50 & 0.29 & @@ & -230.42 & \\
\hline 29 & ACP-1-06-07 x ANDCP-06-07-1 & $\mathrm{P} 3 \times \mathrm{P} 8$ & 240.61 & 1.78 & **@@ & -444.48 & \\
\hline 30 & ACP-1-06-07 x ANDCP-06-07-2 & P3 x P9 & 172.29 & 0.82 & $* *$ & -353.26 & \\
\hline 31 & SKP-84 x VP-1 & P4 x P5 & 204.30 & 1.10 & $* *$ & 552.41 & \\
\hline 32 & SKP-84 x DPC-9 & $\mathrm{P} 4 \times \mathrm{P} 6$ & 355.28 & 0.69 & & 5058.24 & $\#$ \\
\hline 33 & SKP-84 x JP-65 & $\mathrm{P} 4 \times \mathrm{P} 7$ & 410.35 & 2.19 & $*$ & 8714.75 & $\#$ \\
\hline 34 & SKP-84 x ANDCP-06-07-1 & $\mathrm{P} 4 \times \mathrm{P} 8$ & 231.31 & 1.57 & **@@@ & -216.99 & \\
\hline 35 & SKP-84 x ANDCP-06-07-2 & P4 x P9 & 245.62 & 1.51 & **@@@ & -156.38 & \\
\hline 36 & VP-1 x DPC-9 & P5 x P6 & 305.38 & 1.32 & $* *$ & 276.35 & \\
\hline 37 & VP-1 x JP-65 & P5 x P7 & 317.71 & 2.07 & $* *$ & 3304.86 & $\#$ \\
\hline 38 & VP-1 x ANDCP-06-07-1 & $\mathrm{P} 5 \times \mathrm{P} 8$ & 219.79 & 0.46 & & 3580.15 & $\#$ \\
\hline 39 & VP-1 x ANDCP-06-07-2 & P5 x P9 & 182.96 & 0.63 & **@@@ & -264.62 & \\
\hline 40 & DPC-9 x JP-65 & P6 x P7 & 234.49 & 0.64 & & 22812.66 & $\#$ \\
\hline 41 & DPC-9 x ANDCP-06-07-1 & P6 x P8 & 302.89 & 1.23 & $* *$ & 618.40 & \\
\hline 42 & DPC-9 x ANDCP-06-07-2 & P6 x P9 & 291.65 & 2.22 & $* *$ & 5699.24 & \# \\
\hline 43 & JP-65 x ANDCP-06-07-1 & P7 x P8 & 368.75 & 2.52 & $* *$ & 7211.79 & $\#$ \\
\hline 44 & JP-65 x ANDCP-06-07-2 & P7 x P9 & 319.18 & 1.74 & $* *$ & 3020.55 & $\#$ \\
\hline 45 & ANDCP-06-07-1 x ANDCP-06-07-2 & P8 x P9 & 238.05 & 0.04 & & 6236.23 & \# \\
\hline \multirow[t]{2}{*}{46} & GCH-7 & Check & 358.64 & 3.16 & **@@@ & 234.71 & \\
\hline & Hybrids Mean & & 258.70 & & & & \\
\hline
\end{tabular}

***

@,@@

$\#$
Significant at 0.05 and 0.01 percent level, respectively when $\mathrm{H}_{0}$ : $\mathrm{b}=0$

Significant at 0.05 and 0.01 percent level respectively, when $\mathrm{H}_{0}: \mathrm{b}=1$

Significant at 0.05 percent level 
Table.4 Stability parameters for plant height up to base of primary raceme and number of nodes up to base of primary raceme

\begin{tabular}{|c|c|c|c|c|c|c|c|c|c|c|c|}
\hline \multirow[t]{2}{*}{ SN } & \multirow[t]{2}{*}{ Parents / Crosses } & \multirow[t]{2}{*}{ Code } & \multicolumn{5}{|c|}{$\begin{array}{l}\text { Plant height up to base of primary } \\
\text { raceme }\end{array}$} & \multicolumn{4}{|c|}{$\begin{array}{c}\text { Number of nodes up to primary } \\
\text { raceme }\end{array}$} \\
\hline & & & Mean & & $b_{i}$ & $\mathbf{S}^{2} \mathbf{d}_{\mathrm{i}}$ & & Mean & & $b_{i}$ & $\mathbf{S}^{2} \mathbf{d}_{\mathrm{i}}$ \\
\hline 1 & ANDCP-08-01 & P1 & 26.73 & 0.85 & & -6.35 & & 14.96 & 0.45 & & $1.79 \#$ \\
\hline 2 & ANDCP-06-07 & $\mathrm{P} 2$ & 44.29 & 0.87 & & -4.78 & & 16.02 & 0.41 & & 0.30 \\
\hline 3 & ACP-1-06-07 & P3 & 57.44 & -2.15 & & $70.33 \neq$ & \# & 20.33 & 2.38 & $* *$ & $3.06 \#$ \\
\hline 4 & SKP-84 & P4 & 46.20 & 0.97 & ** & -9.52 & & 18.04 & 1.47 & ** & 0.17 \\
\hline 5 & VP-1 & P5 & 35.27 & -1.18 & **@@ & -8.48 & & 12.78 & 2.13 & $*$ & $5.00 \#$ \\
\hline 6 & DPC-9 & P6 & 73.87 & 1.61 & $* *$ & -9.62 & & 14.84 & -0.15 & @ @ & 0.24 \\
\hline 7 & JP-65 & $\mathrm{P} 7$ & 109.47 & 0.47 & **@@ & -12.12 & & 18.04 & 1.11 & $* *$ & -1.20 \\
\hline 8 & ANDCP-06-07-1 & P8 & 45.67 & -2.11 & **@@ & -12.21 & & 15.16 & 1.46 & ** & 0.10 \\
\hline \multirow[t]{2}{*}{9} & ANDCP-06-07-2 & P9 & 43.02 & 0.85 & & 27.82 & & 16.56 & 0.44 & & 0.05 \\
\hline & Parental Mean & & 53.55 & & & & & 16.30 & & & \\
\hline 10 & ANDCP-08-01 x ANDCP-06-07 & $\mathrm{P} 1 \times \mathrm{P} 2$ & 33.16 & 1.25 & & 2.18 & & 14.18 & 0.92 & & 1.17 \\
\hline 11 & ANDCP-08-01 x ACP-1-06-07 & P1 $x$ P3 & 36.51 & 2.28 & **@@ & -8.36 & & 14.67 & 1.31 & & $1.70 \#$ \\
\hline 12 & ANDCP-08-01 x SKP-84 & $\mathrm{P} 1 \times \mathrm{P} 4$ & 36.20 & 0.23 & & 1.54 & & 14.42 & 0.22 & @@ & -0.29 \\
\hline 13 & ANDCP-08-01 x VP-1 & $\mathrm{P} 1 \times \mathrm{P} 5$ & 34.87 & 1.30 & & -4.04 & & 14.40 & 1.31 & $* *$ & 0.11 \\
\hline 14 & ANDCP-08-01 x DPC-9 & P1 x P6 & 75.51 & 0.38 & & -7.44 & & 13.51 & 0.47 & & $1.83 \#$ \\
\hline 15 & ANDCP-08-01 x JP-65 & P1 x P7 & 76.42 & 0.96 & & $43.86 \neq$ & $\#$ & 15.11 & 0.64 & **@@ & -0.53 \\
\hline 16 & ANDCP-08-01 x ANDCP-06-07-1 & P1 x P8 & 36.42 & 1.87 & & 14.53 & & 14.87 & 1.43 & $* *$ & 0.10 \\
\hline 17 & ANDCP-08-01 x ANDCP-06-07-2 & P1 $\times$ P9 & 40.42 & 0.78 & & 20.96 & & 15.78 & 1.09 & $* *$ & -0.30 \\
\hline 18 & ANDCP-06-07 x ACP-1-06-07 & $\mathrm{P} 2 \times \mathrm{P} 3$ & 51.58 & -1.05 & **@@ @ & -11.86 & & 17.29 & 2.13 & **@@ & -0.33 \\
\hline 19 & ANDCP-06-07 x SKP-84 & $\mathrm{P} 2 \times \mathrm{P} 4$ & 38.60 & 1.87 & $*$ & 7.22 & & 16.13 & 0.53 & & 1.11 \\
\hline 20 & ANDCP-06-07 x VP-1 & $\mathrm{P} 2 \times \mathrm{P} 5$ & 31.36 & 0.93 & & 14.10 & & 13.51 & 1.84 & $*$ & $2.26 \#$ \\
\hline 21 & ANDCP-06-07 x DPC-9 & P2 x P6 & 90.22 & 3.00 & **@@ & -12.17 & & 14.53 & 0.15 & @ @ & -0.51 \\
\hline 22 & ANDCP-06-07 x JP-65 & $\mathrm{P} 2 \times \mathrm{P} 7$ & 78.78 & 5.06 & **@@ @ & 12.03 & & 15.22 & 0.66 & **@@ & -0.51 \\
\hline 23 & ANDCP-06-07 x ANDCP-06-07-1 & $\mathrm{P} 2 \times \mathrm{P} 8$ & 46.80 & 2.14 & **@@ @ & -11.44 & & 15.49 & 1.25 & $* *$ & -0.03 \\
\hline 24 & ANDCP-06-07 x ANDCP-06-07-2 & $\mathrm{P} 2 \times \mathrm{P} 9$ & 48.80 & 2.28 & **@@ & -12.03 & & 15.93 & 0.61 & @ @ & 0.05 \\
\hline 25 & ACP-1-06-07 x SKP-84 & $\mathrm{P} 3 \times \mathrm{P} 4$ & 60.42 & -0.70 & & $58.69 \neq$ & $\#$ & 19.84 & 2.89 & **@@ & -0.44 \\
\hline 26 & ACP-1-06-07 x VP-1 & P3 x P5 & 50.22 & 0.57 & & -8.53 & & 17.00 & 1.59 & $* *$ & -0.43 \\
\hline 27 & ACP-1-06-07 x DPC-9 & P3 x P6 & 82.09 & 1.08 & & $60.20 \neq$ & \# & 15.11 & 0.82 & $* *$ & -0.22 \\
\hline 28 & ACP-1-06-07 x JP-65 & P3 x P7 & 122.71 & 4.41 & **@@ @ & 17.74 & & 16.98 & 0.72 & $* *$ & -0.44 \\
\hline 29 & ACP-1-06-07 x ANDCP-06-07-1 & P3 x P8 & 59.11 & 0.49 & **@@ @ & -12.16 & & 17.76 & 1.03 & $* *$ & -0.42 \\
\hline 30 & ACP-1-06-07 x ANDCP-06-07-2 & P3 x P9 & 50.47 & -1.49 & **@@ @ & -12.12 & & 19.16 & 1.72 & **@@ @ & -3.31 \\
\hline 31 & SKP-84 x VP-1 & P4 x P5 & 39.82 & 0.95 & & 20.17 & & 16.71 & 0.99 & $* *$ & 0.15 \\
\hline 32 & SKP-84 x DPC-9 & P4 x P6 & 65.76 & 1.98 & & $94.57 \neq$ & \# & 14.91 & 0.59 & & $3.60 \#$ \\
\hline 33 & SKP-84 x JP-65 & P4 x P7 & 103.51 & 3.85 & **@ & 30.78 & & 17.96 & 0.87 & $* *$ & -0.51 \\
\hline 34 & SKP-84 x ANDCP-06-07-1 & P4 x P8 & 47.82 & 2.52 & **@@ @ & -12.03 & & 16.69 & 1.25 & $*$ & -0.52 \\
\hline 35 & SKP-84 x ANDCP-06-07-2 & P4 x P9 & 48.02 & 2.16 & $*$ & 0.85 & & 16.11 & 1.29 & $*$ & 0.87 \\
\hline 36 & VP-1 x DPC-9 & P5 x P6 & 93.20 & -3.11 & & $600.66 \neq$ & $\#$ & 15.67 & -0.24 & @ & 0.70 \\
\hline 37 & VP-1 x JP-65 & P5 x P7 & 84.04 & 5.52 & **@@ & 19.95 & & 14.11 & 1.02 & $* *$ & -0.38 \\
\hline 38 & VP-1 x ANDCP-06-07-1 & P5 x P8 & 36.71 & -0.60 & & 25.25 & & 13.11 & 2.04 & **@@ & -0.44 \\
\hline 39 & VP-1 x ANDCP-06-07-2 & P5 $x$ P9 & 34.80 & -0.91 & **@@ & -12.09 & & 13.93 & 1.85 & **@@ & -0.52 \\
\hline 40 & DPC-9 x JP-65 & P6 x P7 & 98.07 & 1.26 & $* *$ & -9.88 & & 15.22 & 0.45 & & 2.08 \# \\
\hline 41 & DPC-9 x ANDCP-06-07-1 & P6 $x$ P8 & 88.76 & 0.04 & & $104.82 \neq$ & $\#$ & 15.58 & 0.42 & **@@ & -0.52 \\
\hline 42 & DPC-9 x ANDCP-06-07-2 & P6 x P9 & 80.96 & 0.91 & & -6.31 & & 14.91 & 0.32 & $* *$ & $4.25 \#$ \\
\hline 43 & JP-65 x ANDCP-06-07-1 & P7 x P8 & 106.71 & 0.11 & & 20.02 & & 16.07 & 0.38 & @ & -0.14 \\
\hline 44 & JP-65 x ANDCP-06-07-2 & P7 x P9 & 100.00 & 0.81 & & $36.26 \neq$ & \# & 16.20 & 0.11 & @@ & -0.53 \\
\hline 45 & ANDCP-06-07-1 x ANDCP-06-07-2 & $\mathrm{P} 8 \times \mathrm{P} 9$ & 45.36 & -0.12 & & $48.17 \neq$ & $\#$ & 16.18 & 0.65 & & 0.45 \\
\hline \multirow[t]{2}{*}{46} & GCH-7 & Check & 85.04 & 2.82 & $* *$ & 19.95 & & 15.98 & 1.00 & $*$ & 0.47 \\
\hline & Hybrids Mean & & 63.22 & & & & & 15.68 & & & \\
\hline
\end{tabular}

$*$,** Significant at 0.05 and 0.01 percent level, respectively when $\mathrm{H}_{0}: \mathrm{b}=0$ @, @ @ Significant at 0.05 and 0.01 percent level respectively, when $\mathrm{H}_{0}$ : b=1 \# Significant at 0.05 percent level 
Table.5 Stability parameters for days to $50 \%$ flowering of primary raceme and days to $50 \%$ maturity of primary raceme

\begin{tabular}{|c|c|c|c|c|c|c|c|c|c|c|c|c|}
\hline \multirow{3}{*}{$\begin{array}{c}\text { SN } \\
\\
1\end{array}$} & \multirow[t]{3}{*}{ Parents / Crosses } & \multirow{3}{*}{\begin{tabular}{|c|} 
Code \\
P1 \\
\end{tabular}} & \multicolumn{5}{|c|}{$\begin{array}{c}\text { Days to } 50 \% \text { flowering of primary } \\
\text { raceme }\end{array}$} & \multicolumn{5}{|c|}{$\begin{array}{c}\text { Days to } 50 \% \text { maturity of primary } \\
\text { raceme }\end{array}$} \\
\hline & & & \multirow{2}{*}{$\begin{array}{c}\text { Mean } \\
64.00\end{array}$} & \multicolumn{2}{|c|}{$\mathbf{b}_{\mathbf{i}}$} & \multicolumn{2}{|l|}{$S^{2} d_{i}$} & \multirow{2}{*}{$\begin{array}{r}\text { Mean } \\
141.44\end{array}$} & \multicolumn{2}{|c|}{$\mathbf{b}_{\mathbf{i}}$} & \multicolumn{2}{|l|}{$\mathbf{S}^{2} \mathbf{d}_{\mathbf{i}}$} \\
\hline & & & & 1.17 & $* *$ & 35.95 & $\#$ & & 0.61 & **@ & -0.41 & \\
\hline 2 & ANDCP-06-07 & P2 & 65.56 & 1.17 & ** & 1099 & & 136.67 & 1.51 & 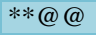 & -6.47 & \\
\hline 3 & ACP-1-06-07 & P3 & 78.33 & 1.23 & ** & 29.19 & \# & 148.78 & 0.83 & & 38.86 & \\
\hline 4 & SKP-84 & P4 & 67.00 & 1.05 & $* *$ & -4.11 & & 138.11 & 0.42 & **@@ & -5.10 & \\
\hline 5 & VP-1 & P5 & 60.56 & 0.40 & **@@ & -4.15 & & 128.44 & 0.74 & **@ & -4.52 & \\
\hline 6 & DPC-9 & P6 & 70.44 & 1.30 & $* *$ & 18.22 & $\#$ & 134.56 & 1.51 & **@@ & -5.21 & \\
\hline 7 & JP-65 & P7 & 73.22 & 1.41 & **@ & 4.25 & & 156.44 & 0.94 & ** & -2.20 & \\
\hline 8 & ANDCP-06-07-1 & P8 & 59.33 & 0.64 & **@@ & -0.08 & & 128.00 & 1.38 & $* *$ & 2.66 & \\
\hline \multirow[t]{2}{*}{9} & ANDCP-06-07-2 & P9 & 65.67 & 1.15 & $* *$ & -2.39 & & 142.67 & 1.06 & $*$ & $38.05 \neq$ & $\#$ \\
\hline & Parental Mean & & 67.12 & & & & & 139.46 & & & & \\
\hline 10 & ANDCP-08-01 x ANDCP-06-07 & P1 x P2 & 56.67 & 0.91 & $* *$ & -0.76 & & 128.67 & 0.79 & **@@ & -5.40 & \\
\hline 11 & ANDCP-08-01 x ACP-1-06-07 & P1 x P3 & 62.67 & 0.61 & **@@ & -0.53 & & 135.00 & 0.85 & $* *$ & -4.64 & \\
\hline 12 & ANDCP-08-01 x SKP-84 & P1 x P4 & 63.00 & 0.94 & $* *$ & -3.99 & & 128.11 & 0.90 & **@ & -6.08 & \\
\hline 13 & ANDCP-08-01 x VP-1 & P1 x P5 & 64.78 & 1.01 & $* *$ & -4.28 & & 136.44 & 0.91 & $*$ & $32.64 \neq$ & $\#$ \\
\hline 14 & ANDCP-08-01 x DPC-9 & P1 x P6 & 59.56 & 0.87 & $* *$ & 2.69 & & 130.67 & 1.60 & **@@ & -4.58 & \\
\hline 15 & ANDCP-08-01 x JP-65 & P1 x P7 & 58.78 & 0.63 & **@@ & -0.73 & & 134.44 & 0.97 & $*$ & $30.61 \neq$ & $\#$ \\
\hline 16 & ANDCP-08-01 x ANDCP-06-07-1 & P1 x P8 & 60.33 & 0.91 & ** & 20.08 & \# & 126.89 & 0.49 & & 9.85 & \\
\hline 17 & ANDCP-08-01 x ANDCP-06-07-2 & P1 x P9 & 64.67 & 1.23 & **@@ & -4.20 & & 139.33 & 1.04 & $* *$ & -1.37 & \\
\hline 18 & ANDCP-06-07 x ACP-1-06-07 & $\mathrm{P} 2 \times \mathrm{P} 3$ & 66.22 & 1.00 & $* *$ & 10.74 & & 135.33 & 1.02 & $*$ & $46.57 \neq$ & $\#$ \\
\hline 19 & ANDCP-06-07 x SKP-84 & $\mathrm{P} 2 \times \mathrm{P} 4$ & 65.00 & 1.09 & $* *$ & 1.66 & & 131.67 & 1.29 & $* *$ & $20.26 \neq$ & $\#$ \\
\hline 20 & ANDCP-06-07 x VP-1 & $\mathrm{P} 2 \times \mathrm{P} 5$ & 59.33 & 0.85 & **@@ & -4.63 & & 131.00 & 1.39 & **@ & 0.07 & \\
\hline 21 & ANDCP-06-07 x DPC-9 & P2 x P6 & 61.44 & 0.99 & $* *$ & -4.03 & & 142.56 & 0.71 & & $47.12 \neq$ & $\#$ \\
\hline 22 & ANDCP-06-07 x JP-65 & $\mathrm{P} 2 \times \mathrm{P} 7$ & 62.78 & 1.01 & $* *$ & -2.22 & & 139.78 & 0.92 & $* *$ & -0.78 & \\
\hline 23 & ANDCP-06-07 x ANDCP-06-07-1 & P2 x P8 & 62.22 & 0.93 & $* *$ & -3.94 & & 132.67 & 0.74 & & $60.06 \neq$ & $\#$ \\
\hline 24 & ANDCP-06-07 x ANDCP-06-07-2 & $\mathrm{P} 2 \times \mathrm{P} 9$ & 63.11 & 1.21 & **@@ & -4.64 & & 131.33 & 0.98 & $* *$ & -1.07 & \\
\hline 25 & ACP-1-06-07 x SKP-84 & P3 x P4 & 72.44 & 0.53 & *@ & 9.30 & & 144.33 & 0.82 & $* *$ & 2.96 & \\
\hline 26 & ACP-1-06-07 x VP-1 & P3 x P5 & 63.89 & 0.98 & $* *$ & 5.29 & & 131.11 & 0.63 & **@@ & -5.36 & \\
\hline 27 & ACP-1-06-07 x DPC-9 & P3 x P6 & 61.89 & 0.68 & **@@ & -3.92 & & 127.11 & 0.93 & $* *$ & -5.56 & \\
\hline 28 & ACP-1-06-07 x JP-65 & P3 x P7 & 67.00 & 1.19 & $* *$ & 7.19 & & 143.89 & 0.94 & $* *$ & 3.75 & \\
\hline 29 & ACP-1-06-07 x ANDCP-06-07-1 & P3 x P8 & 67.00 & 1.09 & **@@ & -4.63 & & 142.33 & 0.39 & & $30.67 \neq$ & $\#$ \\
\hline 30 & ACP-1-06-07 x ANDCP-06-07-2 & P3 x P9 & 74.33 & 1.02 & $* *$ & 11.14 & & 144.78 & 0.64 & **@@ & -6.52 & \\
\hline 31 & SKP-84 x VP-1 & P4 x P5 & 65.89 & 1.03 & $* *$ & 2.72 & & 135.00 & 0.48 & **@@ & -6.56 & \\
\hline 32 & SKP-84 x DPC-9 & P4 x P6 & 62.67 & 1.12 & **@@ & -4.39 & & 128.78 & 1.13 & $* *$ & -0.21 & \\
\hline 33 & SKP-84 x JP-65 & P4 x P7 & 66.89 & 1.17 & **@@ & -3.95 & & 137.33 & 0.85 & $* *$ & -4.64 & \\
\hline 34 & SKP-84 x ANDCP-06-07-1 & P4 x P8 & 62.22 & 1.15 & $* *$ & 0.28 & & 130.33 & 1.08 & $* *$ & -4.49 & \\
\hline 35 & SKP-84 x ANDCP-06-07-2 & P4 x P9 & 63.44 & 1.01 & $* *$ & -2.22 & & 127.22 & 1.16 & $* *$ & 0.38 & \\
\hline 36 & VP-1 x DPC-9 & P5 x P6 & 63.67 & 0.96 & **@@ & -4.64 & & 135.56 & 0.76 & & $32.36 \neq$ & $\#$ \\
\hline 37 & VP-1 x JP-65 & P5 x P7 & 60.33 & 0.93 & $* *$ & 15.06 & $\#$ & 131.67 & 1.80 & **@@ & 1.31 & \\
\hline 38 & VP-1 x ANDCP-06-07-1 & P5 x P8 & 55.56 & 0.66 & **@@ & -3.64 & & 122.67 & 1.05 & $* *$ & -4.05 & \\
\hline 39 & VP-1 x ANDCP-06-07-2 & $\mathrm{P} 5 \times \mathrm{P} 9$ & 59.89 & 0.67 & **@@ & -2.02 & & 128.33 & 1.03 & $* *$ & 15.23 & \\
\hline 40 & DPC-9 x JP-65 & P6 x P7 & 64.33 & 1.12 & $* *$ & -0.16 & & 139.11 & 1.37 & $* *$ & 3.96 & \\
\hline 41 & DPC-9 x ANDCP-06-07-1 & $\mathrm{P} 6 \times \mathrm{P} 8$ & 65.00 & 1.21 & **@@ & -3.23 & & 132.67 & 0.91 & $*$ & $24.14 \neq$ & $\#$ \\
\hline 42 & DPC-9 $\mathbf{x}$ ANDCP-06-07-2 & P6 x P9 & 62.00 & 0.95 & $* *$ & 26.62 & $\#$ & 126.89 & 0.68 & **@ & 1.61 & \\
\hline 43 & JP-65 x ANDCP-06-07-1 & P7 x P8 & 66.22 & 1.42 & $* *$ & 13.12 & & 136.11 & 1.37 & $* *$ & 17.87 & \\
\hline 44 & JP-65 x ANDCP-06-07-2 & P7 x P9 & 67.22 & 1.47 & **@@ & -0.18 & & 138.11 & 1.63 & **@ & 7.51 & \\
\hline 45 & ANDCP-06-07-1 x ANDCP-06-07-2 & P8 x P9 & 66.22 & 1.17 & $* *$ & -2.87 & & 133.67 & 1.75 & **@ & 12.26 & \\
\hline \multirow[t]{2}{*}{46} & GCH-7 & Check & 63.00 & 0.77 & $* *$ & -0.50 & & 132.22 & 1.02 & $*$ & $31.74 \neq$ & $\#$ \\
\hline & Hybrids Mean & & 63.56 & & & & & 133.87 & & & & \\
\hline
\end{tabular}

*, ** Significant at 0.05 and 0.01 percent level, respectively when $\mathrm{H}_{0}$ : $\mathrm{b}=0$

@, @ @ Significant at 0.05 and 0.01 percent level respectively, when $\mathrm{H}_{0}$ : b=1

\# Significant at 0.05 percent level 
From present study, it was revealed that none of the crosses was found consistent for its performance over environments for all the attributes, which might be because of sensitivity of parental genes to environmental differences. Such interaction result in change of relative ranking of different genotypes and also alters magnitude of difference between genotypes, which create problem for plant breeders in making proper assessment of genotypes, when the same are tested over varied environments (Comstock and Moll, 1963). Therefore, more vigours testing/ evaluation of the crosses in an array of environments is suggested, environmental specific crosses has been identified with high heterotic and sca effects, and available gene pool may be strengthened with the inclusion of more diverse source of parental genotypes.

\section{References}

Allard, E. W. and Bradshaw, A. D. (1964). Implications of genotype and environment interactions in applied plant breeding. Crop Sci., 4: 503-07.

Anonymous (2019). Annual Report, Castor 2018-19. Directorate of Oilseed Research, Rajendranagar, Hyderabad, 500030

Chaudhari, K. N. (2006). Diallel analysis for seed yield and wilt resistance in castor (Ricinus communis L.). Ph. D. Thesis
(Unpublished) submitted to S. D. Agricultural University, Sardar krushinagar (Gujarat).

Comstock, R. E. and Moll, R. H. (1963). Genotype-environment interactions. In: Statistical genetics and plant breeding (Ed. Hanson H.D. and Robinson H.E.) NAS-NRC Publ.982. Washington DC. pp. 164-197.Eberhart, S. A. and Russell, W. A. (1966). Stability parameters for comparing varieties. Crop Sci., 6: 3640.

Patel, A. R. (2009) Genetic analysis of seed yield and component characters over environments in castor (Ricinus communis L.). Ph.D. Thesis (Unpublished) submitted to Anand Agricultural University, Anand (Gujarat).

Sasidharan, N. (2005). Genetic analysis for yield and quality in castor (Ricinus communis L.) under diverse environments. Ph.D Thesis (Unpublished) submitted to Anand Agricultural University, Anand (Gujarat).

Solanki, S. S. and Joshi, P. (2003). Phenotypic stability of hybrids and parents for seed yield and its components in castor (Ricinus communis L.). J. Oilseeds Res., 20 (1): 45-47.

\section{How to cite this article:}

Aher, A. R., M. S. Kamble, M. S. Mote, A. G. Bhoite and Shinde, S. R. 2020. Stability Analysis of Pistillate x Pistillate based Hybrids and their Parents for Seed Yield in Castor (Ricinus communis L.). Int.J.Curr.Microbiol.App.Sci. 9(08): 1324-1332. doi: https://doi.org/10.20546/ijcmas.2020.908.150 